Available online at GSC Online Press Directory

GSC Biological and Pharmaceutical Sciences

e-ISSN: 2581-3250, CODEN (USA): GBPSC2

Journal homepage: https://www.gsconlinepress.com/journals/gscbps

(RESEARCH ARTICLE)

\title{
Prescription profile for urgent biochemical analyzes at the biochemistry laboratory in Antananarivo
}

\author{
Miora Koloina Ranaivosoa 1, ${ }^{*}$, Tina Rakotoniaina ${ }^{2}$, Alain Ntoezara ${ }^{3}$, Saraha Rabeherisoa ${ }^{4}$, Olivat Rakoto \\ Alson ${ }^{5}$ and Andry Rasamindrakotroka ${ }^{6}$ \\ ${ }^{1}$ Biologist, Paraclinical Training and Biochemistry Research Unit of The Joseph Ravoahangy Andrianavalona, University \\ of Antananarivo, Madagascar \\ ${ }^{2}$ Médical Biology Student, Paraclinical Training and Biochemistry Research Unit of The Joseph Ravoahangy \\ Andrianavalona, University of Antananarivo, Madagascar \\ ${ }^{3}$ Biologist, Laboratory Unit of University Hospital Center Tanambao I Antsiranana, Madagascar \\ ${ }^{4}$ Doctor, National Malaria Control Program in Androhibe, Madagascar \\ 5 Professor of Biological Haematology, Medical Biology Department of the Faculty of Medicine Antananarivo, Madagascar \\ ${ }^{6}$ Professor of Immunology, Laboratory of Training and Research in Medical Biology, University of Antananarivo, \\ Madagascar.
}

Publication history: Received on 27 November 2020; revised on 05 December 2020; accepted on 07 December 2020

Article DOI: https://doi.org/10.30574/gscbps.2020.13.3.0396

\begin{abstract}
The extreme emergency in biochemistry matches with the detection and/or monitoring of an acute or chronic pathological condition, at the root of a vital failure or a systemic imbalance deleterious for the organism. This study aims at determining the prescription profile of biochemical tests prescribed on call at the Paraclinical Training and Biochemistry Research Unit of the Joseph Ravoahangy Andrianavalona. The specific objectives are to determine the proportion of tests prescribed by the departments qualified as "urgent" and to determine the frequency of request for each biochemical parameter prescribed during on-call duty.

This is a descriptive retrospective study over a period of 5 Months, from November 2016 to March 2017 . All the biochemical testing prescribed during the study period and carried out in the laboratory of Joseph Ravoahangy Andrianavalona University Hospital Center were used.

Out of the 595 biochemical testing prescribed during on-call period, 588 (98.82\%) were included in the study.

Two hundred and seventy-four (46.60\%) of these testing were prescribed by departments classified as "non-urgent" and $314(53.40 \%)$ by departments classified as "urgent". We found that non-urgent parameters were prescribed such as lipid status and HbA1c at less than $2 \%$. Creatinine was prescribed at $99.60 \%$, followed by blood ionogram at $65.99 \%$ and urea at $40.48 \%$.
\end{abstract}

Biochemical parameters prescribed during on-call periode are limited and that biologists must develop a list of feasible biochemical parameters on call periode for better management of patiens as soon as possible.

Keywords: Biochemistry; Emergency; Prescription; Profile

\footnotetext{
${ }^{*}$ Corresponding author: Miora Koloina Ranaivosoa

Mailing address: Paraclinical Training and Biochemistry Research Unit of the Joseph Ravoahangy Andrianavalona, Antananarivo, Madagascar.

Copyright (@ 2020 Author(s) retain the copyright of this article. This article is published under the terms of the Creative Commons Attribution Liscense 4.0.
} 


\section{Introduction}

The extreme emergency in biochemistry matches with the detection and/or monitoring of an acute or chronic pathological condition, at the root of a vital failure or a systemic imbalance deleterious for the organism. The outcomes of emergency biochemical examinations can also be used as a basis for the subsequent follow-up of patients, for example after the initiation of therapy. Biochemistry emergencies can also be organizational, helping to regulate patient flows in an emergency department for example, as it promotes downstream orientation (return home, observation or hospitalization). The case of an emergency request for a cardiac marker such as troponin is a good illustration of this situation [1]. Most biochemical tests called emergency tests are also biological emergencies because the shelf life of whole blood tests is limited (potassium, glucose...) [2]. This study aims at determining the prescription profile of biochemical tests prescribed on call at the Biochemistry UPFR of the hospital CHU JRA. The specific objectives are to determine the proportion of tests prescribed by the departments qualified as "urgent" and to determine the frequency of request for each biochemical parameter prescribed during on-call duty.

\section{Material and methods}

This is a descriptive retrospective study over a period of 5 Months, from November 2016 to March 2017 . All the biochemical testing prescribed during the study period and carried out in our laboratory were used.

All the blood biochemical testing received during the on-call period (from 5 p.m. to 8 a.m. during the week, during Saturday and Sunday during the 48-hour on-call period and on holidays) were included in the study. All non-compliant, incomplete testing, urinary or other fluid analyses were excluded from this study. Data were entered and processed on Microsoft Office Excel® 2013.

\section{Results}

Out of the 595 biochemical testing prescribed during on-call period, 588 (98.82\%) were included in the study. Seven records were excluded, 4 for incomplete records and 3 for parameters other than blood. The mean age of all patients was 39.33 years with extremes of 1 day and 91 years. The sex ratio was 0.91 with a female predominance. Two hundred and seventy-four (46.60\%) of these testing were prescribed by departments classified as "non-urgent" and 314 $(53.40 \%)$ by departments classified as "urgent". The latter were represented by the different resuscitations and emergencies, namely: emergency department (21.09\%), surgical resuscitation (17.18\%), medical resuscitation (9.35\%), emergency resuscitation (3.23\%), nephrological resuscitation $(1.70 \%)$ and Befelatanana maternity resuscitation $(0.85 \%)$.

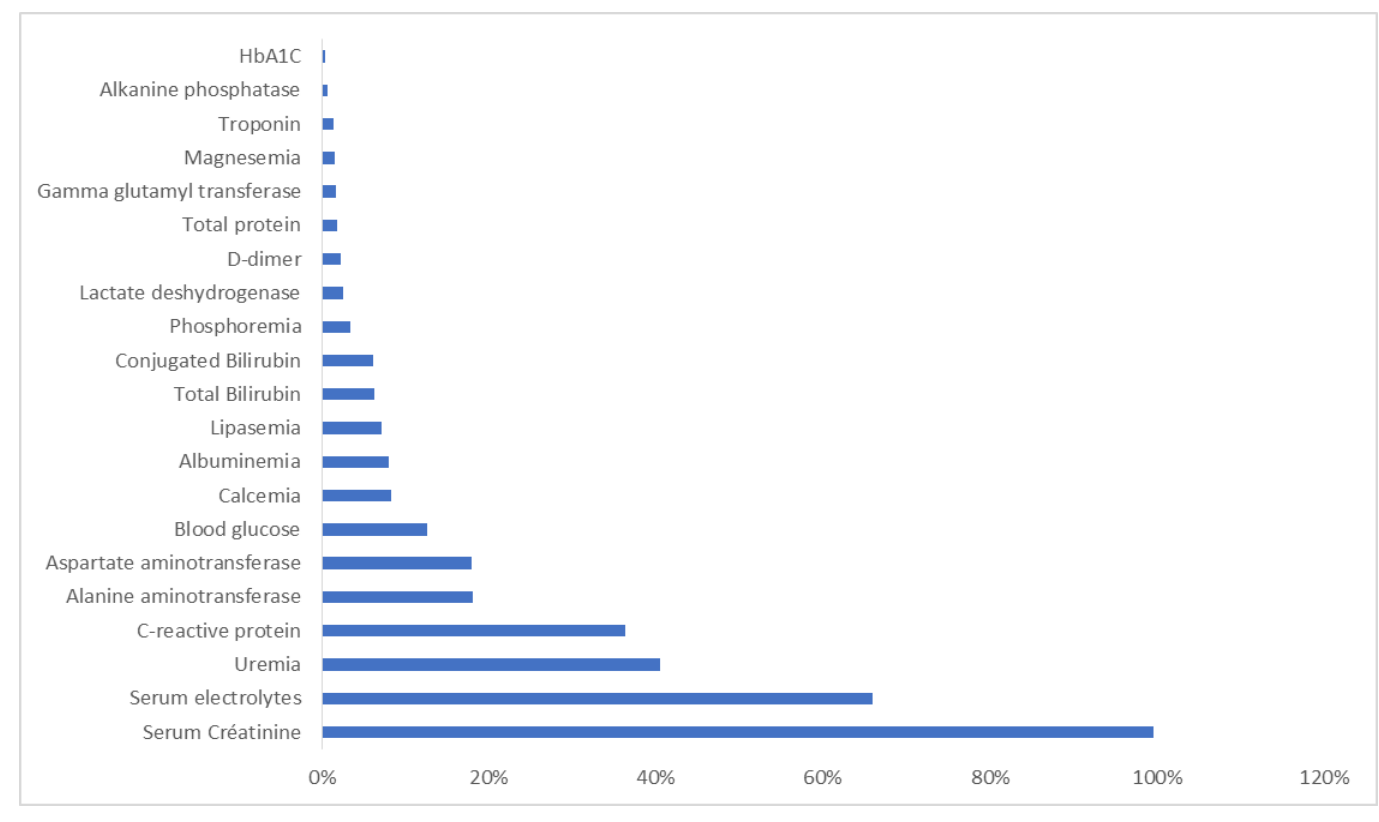

Figure 1 Frequency of biochemical parameters prescribed during on-call period 
The departments qualified as "Non-urgent" were represented by the Befelatanana Maternity Department with $12.59 \%$, followed by Oncology (8.67\%), Urology (3.40\%), Thoracic Surgery (3.06\%), Visceral Surgery (2.89\%), Gastroenterology $(2.72 \%)$, Neurosurgery (2.21\%), and Traumatology $(2.0 \%), 04 \%)$, Cardiovascular Surgery (1.87\%), Child Surgery $(1.36 \%)$, Fenoarivo University Hospital (1.36\%), and Nephrology with $1.02 \%$. Finally, other departments such as Ophthalmology, Cardiology, Rheumato-dermatology, Endocrinology, Stomatology, and Pediatrics keep the last places with percentages between 0 and 0.5 and $1 \%$.

\section{Discussion}

Forty-seven-point eleven percent of biochemical testing conducted during on-call period were prescribed by departments classified as "non-urgent", while department classified as "urgent" accounted for 52.89\% of biochemical testing during on-call period. The latter were represented by the different resuscitations and emergencies, namely: emergency department (21.09\%), surgical resuscitation (17.18\%), medical resuscitation (9.35\%), emergency resuscitation (3.23\%), nephrology resuscitation (1.70\%) and Befelatanana maternity resuscitation (0.85\%). All of these departments except Befelatanana Maternity Resuscitation are located in the same facility as the laboratory, which explains the high rate of prescriptions from departments qualified as "urgent".

The most frequent prescription during the on-call period was creatinine with a rate of $99.66 \%$, a finding similar to the study carried out by the team of Assiya El Kettani et al. [3]. The blood ionogram was prescribed with a rate of $65.99 \%$. It is a parameter that allows the evaluation of a hydroelectrolytic imbalance, and is useful in emergency situations to guide the diagnosis when there are signs of a hydroelectrolytic disorder (muscle weakness, paralysis, behavioral disorders, convulsions) or in circumstances that favor the occurrence of ionic imbalances (digestive disorders, polytrauma, heart or kidney failure, diabetes, fever, etc.) [1]. In intensive care units, these parameters are also part of the periodic biological monitoring of patients, which explains the high frequency of prescriptions. Urea was requested in 3rd place at $40.48 \%$. Creatinine and urea are part of the parameters urgently prescribed [list of tests prescribed during on-call period] and are used to evaluate renal function [3]. According to some learned societies, a list of tests that can be prescribed on an emergency basis should be proposed. Therefore, one of the roles of the biologist in the appropriateness of prescribing urgent tests is to establish at a first glance a list of tests that can be performed "on an emergency basis". This list is established based on pre-analytical and analytical criteria, diagnostic value of the tests and the impact of these tests on patient management $[4,5]$. The laboratory of the Biochemistry UPFR of the hospital CHU JRA has a list of tests that can be prescribed urgently.

In our case, the lipid profile, GGT, magnesium, PAL, HbA1C were prescribed less than $2 \%$. In fact, these parameters are not on our list of tests that can be urgently prescribed, and most prescribing physicians have already been made aware of these tests available during on-call period, which explains this low frequency. Compared to the list proposed by the Société Française de Biologie Clinique [1], GGT and PAL are among the tests performed when they are on call.m

Troponin in this study was prescribed at only 1.36\%, this low prescribing rate may be related to its high cost compared to other parameters during on-call period

\section{Conclusion}

Biochemical parameters prescribed during on-call period are limited and that biologists must develop a list of feasible biochemical parameters on call period for better management of patient as soon as possible.

\section{Compliance with ethical standards}

\section{Acknowledgments}

We would like to thank all the staff of the laboratory of University Hospital of Ravoahangy Andrianavalona. Similarly, we would like to express our gratitude to the director of establishment for authorizing us to carry out this study.

\section{Disclosure of conflict of interest}

The Author declare no conflict of interest. 


\section{References}

[1] Vaubourdolle M, Alvarez JC, Barbé F, Beaudeux JL, Boissier E, Caillon H, Chatron P, Guillou ML, Mailloux A. SFBC guidelines on critical care testing. Ann Biol Clin. 2016; 74(2): 130-55.

[2] Challine D, Dhondt JL, Szymanowicz A; les membres du sous-groupe pré-analytique - Groupe de travail SFBC "Accreditation of medical biology laboratories" (coordinator M. Vaubourdolle). Recommendations for the urgent review process. Ann Biol Clin. 2010; 68(Hors-série no 1): 147-54.

[3] Assiya ElK, Mohammed ElA, Nabiha K. Relevance of prescriptions for urgent biochemical analyzes at Casablanca Ibn Rochd University Hospital. International Journal of Innovation and Applied Studies. 2018; (24): 1658-63.

[4] Zankera.C, Bezeaudb A, Guimontb MC, Denninger MH, Breaud N, Beauneet S et al. Evaluate the correct prescription of biological examinations in an emergency department. J Eur. 2007; 20(1): 14-15.

[5] Bilbault P, Hane MA, Berna C, Duja C, Mihalcea M, Steinmetz JP et al. Control of prescriptions for biological examinations in emergency rooms: example of amylase-lipases. J Eur. 2009; 22S: 44-48. 